\title{
Una noria gótica en el claustro de la Catedral de Toledo ${ }^{*}$
}

\author{
Amalia M $\mathrm{M}^{\mathrm{a}}$ Yuste GalÁN \\ Universidad Complutense de Madrid \\ amaliayuste@gmail.com \\ Jean PASSINI \\ Centre National de la Recherche Scientifique - UMR 8558 \\ jean.passini@ehess.fr
}

\begin{abstract}
RESUMEN
Tras la observación y análisis de una estrecha cámara, situada bajo la panda sur del claustro, y su relación con el resto de construcciones, proponemos que la noria de la que se habla en el siglo XV estaba instalada en esta original estructura rectangular que se comunica con el antiguo aljibe de la mezquita mayor toledana.

La importancia de esta máquina para sacar agua se refleja en los numerosos asientos, conservados en los Libros de Obra y Fábrica, que citan sus reparaciones en uno de los momentos de mayor actividad constructiva en la Catedral, el primer tercio del siglo XV. Durante esos años se trabajaba en la elevación de la Torre Mayor, de la Torre del Reloj y del claustro. El estudio de las construcciones conservadas en el claustro junto a la documentación medieval del Archivo Capitular pone de manifiesto una compleja red de recuperación, almacenaje y aprovechamiento del agua en el gran edificio catedralicio. Un complejo y eficaz sistema en el que destaca la pervivencia de antiguas estructuras de la mezquita y su adaptación al nuevo edificio gótico.
\end{abstract}

Palabras clave: Catedral de Toledo, arquitectura, suministro de agua, ingeniería hidráulica, noria.

\begin{abstract}
After observing and analyzing the narrow chamber, located under the south side of the cloister, and its relationship to other buildings, we propose that the waterwheel of which is spoken in the fifteenth century is the one raised in this original rectangular structure that communicates with the old cistern of the major mosque of Toledo.

The importance of this machine to obtain water is reflected in the numerous entries, preserved in the "Books of Work and Factoring", mentioning its repairs in one of the major constructive times in the Cathedral, the first third of the fifteenth century. During those years they worked in the raising of the Tower of the Bell, the Tower of the Clock and the Cloister. The research of the preserved buildings in the cloister together with the medieval documents of the Chapter Archive reveals the complex system of recovery, storage and use of water in the great cathedral building. It's a complex and an effective system for utilizing water, stressing the survival of the old structures of the mosque and its adaptation to the new Gothic building.
\end{abstract}

Keywords: Cathedral of Toledo, architecture, water supply, hydraulic engineering, waterwheel. 


\section{Introducción}

La situación del peñón toledano obligó a todos los pueblos que lo habitaron a lo largo de la historia a buscar soluciones para poder abastecerse de agua. Cada civilización utilizó los conocimientos de la anterior para recoger, almacenar y suministrar agua a su población ${ }^{2}$.

De la ciudad romana de Toledo se conservan abundantes testimonios de su complejo sistema hidráulico: cisternas, alcantarillas, cloacas, una presa y los restos de un acueducto ${ }^{3}$. Posteriormente, con la llegada del Islam, la cultura del agua se reflejó en un arte en el que las estructuras hidráulicas se construyeron en palacios, jardines, casas y baños. El significado del agua, como elemento sagrado y signo de pureza, marcó en gran medida la arquitectura musulmana. En los patios de las mezquitas siempre había una fuente donde lavarse antes de dirigirse a la oración y, en muchas ocasiones, junto a ellas se levantaron edificios con baños, fuentes y letrinas ${ }^{4}$. Para poder suministrar agua a estos edificios se construían depósitos, y era habitual la presencia de aljibes bajo los patios de las mezquitas 5 .

En el claustro de la Catedral de Toledo se mantienen dos estructuras hidráulicas: un aljibe y una cámara muy alta de forma oblonga, que corresponden a dos períodos constructivos diferentes. Apoyándonos en la observación directa de ambos espacios y en la documentación medieval de Obra y Fábrica de la Catedral, hemos tratado de entenderlas y explicarlas.

\section{Descripción del aljibe}

Bajo la panda sur del claustro de la Catedral de Toledo se conserva un gran aljibe al que hoy se accede a través de la lumbrera de una de las bóvedas que lo cubren. El aljibe ocupa el tramo central de la crujía y los dos siguientes tramos hacia el este, sin llegar al pilar del ángulo del claustro (fig. 1). En total, tres de los pilares

2 Ante el reto que supuso la escasez de agua en la península y las respuestas paralelas, y satisfactorias, aportadas por diferentes culturas, Pavón Maldonado plantea la posibilidad de una continuidad romano-árabe en las soluciones al problema. B. PAVÓN MALDONADO, Tratado de Arquitectura Hispanomusulmana, t. I Agua, Madrid, 1990, p. 15.

3 F. ARANDA ALONSO, J. CARROBLES SANTOS y J.L. ISABEL SÁNCHEZ, El sistema hidráulico romano de abastecimiento a Toledo, Toledo, 1997, pp. 330-333.

4 El midá de la Mezquita de Granada era un edificio con baños, letrinas, pilas y fuentes para las abluciones. C. VÍLCHEZ VÍLCHEZ y A. ORIHUELA UZAL, "Aljibes públicos de la Granada musulmana", Arqueología Medieval Española, t. III, Madrid, 1987, pp. 231-237.

5 Los aljibes aparecen en una proporción significativa vinculados a las mezquitas. Ejemplos de aljibes musulmanes se conservan en las de Sevilla, Granada y Córdoba. La vinculación entre mezquita y aljibe garantizaba al barrio cierta autonomía, al dotarlo con suministro propio de agua y, además, su ubicación en un espacio sagrado protegía la construcción ante posibles conflictos. A. ORIHUELA UZAL y C. VÍLCHEZ VÍLCHEZ, Aljibes públicos de la Granada islámica, Granada, 1991, p. 51; C. TRILLO SAN JOSÉ, "El agua en las ciudades andalusíes: Madîna Garnāţa y su área periurbana (siglos XI-XV)", Musulmanes y Cristianos frente al agua en las ciudades medievales, Santander, 2008, pp. 103-124. 
del claustro atraviesan la cubierta del depósito, y bajan unos siete metros hasta encontrar el suelo firme donde se asientan sus fundamentos que, como veremos, ocupan parte del aljibe.

De las zonas accesibles ${ }^{6}, \mathrm{y}$ de lo que a través de ellas se logra ver, se deduce que el depósito tiene una planta rectangular, y está dividido en tres salas sub-cuadradas cubiertas por bóvedas baídas de ladrillo. Las paredes son de mampostería recubierta con una capa de mortero hidráulico que impermeabiliza también las cubiertas.

La sala oeste mide $5,80 \times 5,60$ metros, que sumado a las otras dos salas, de las mismas dimensiones, permite calcular una superficie de,

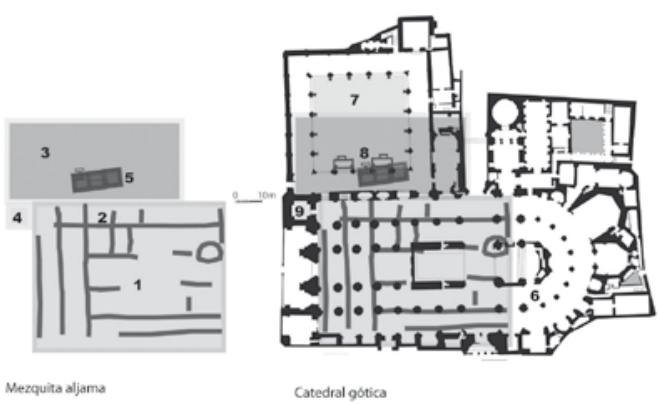

Fig. 1. De la Mezquita a la Catedral. Superposición de las plantas: 1. Planta de la mezquita aljama. 2. Cimentación de las naves. 3. Patio. 4. Alminar. 5. Aljibe. 6. Planta de la Catedral. 7. Claustro. 8. Pozo de la noria. 9. Torre. aproximadamente, $100 \mathrm{~m}^{2}$; con una capacidad, hasta el arranque de la bóveda, de $165 \mathrm{~m}^{3} \mathrm{y}$, superando el tramo recto, pero sin llegar a su cota máxima de, aproximadamente, $390 \mathrm{~m}^{3}$. La altura hasta el centro de la bóveda es de poco más de 5 metros; en este punto se abren unas "chimeneas" de planta cuadrada que llegan hasta al suelo del claustro, de una altura de casi dos metros. Las bóvedas se apoyan sobre los muros perimetrales y los tramos se comunican entre sí a través de arcos de medio punto ${ }^{7}$. El suelo es de losas de barro cocido y todo el interior está recubierto con mortero, probablemente almagre, para impermeabilizarlo ${ }^{8}$. Sus características formales y sus dimensiones convierten el aljibe en un verdadero depósito de acumulación y reparto9.

De las tres salas conocidas, en la situada en el centro se levantó una pared en el lado occidental, hasta una altura de unos dos metros, desde el pilar gótico hasta el muro del propio aljibe, para poder separar el agua almacenada en la otra sala. En

6 La falta de medios para llevar a cabo una completa inspección del aljibe nos obliga a plantear una descripción aproximada de su trazado, así como una hipótesis de trabajo sobre su origen.

7 En Granada se ha llevado a cabo una clasificación tipológica de los aljibes hispanomusulmanes atendiendo al trazado de sus plantas y a los tipos de bóvedas empleadas en sus cubiertas. C. VÍLCHEZ VÍLCHEZ y A. ORIHUELA UZAL, op. cit., 1987, p. 236.

8 El revestimiento hidráulico de color rojo, almagre, era común en los aljibes hispanomusulmanes.

9 El aljibe de la Mezquita Mayor de Granada, datado en el siglo XI, tiene una capacidad de $157 \mathrm{~m}^{3}$. El conocido como "El Viejo", de la misma ciudad, $300 \mathrm{~m}^{3}$. El de la mezquita de Córdoba, mandado construir por Almanzor, tiene una capacidad máxima de $390 \mathrm{~m}^{3}$. L. TORRES BALBÁS, "La Mezquita Mayor de Granada”, Obra Dispersa I. Al-Andalus. Crónica de la España Musulmana, 3, Madrid, 1982, pp. 88-111; B. PAVÓN MALDONADO, op. cit., 1990, p. 20; y C. TRILLO SAN JOSÉ, “Aljibes y Mezquitas en Madîna Garnāţa (siglos XI-XV): Significado Social y Espacial", Espacios de Poder y Formas Sociales en la Edad Media, Salamanca, 2007, pp. 323-324. 
la base se abrió un agujero, junto al que permanece una gran piedra que pudo ser utilizada como tapón. Estos añadidos permitían controlar el nivel de cada sala y las presiones ejercidas por el volumen de agua allí almacenado.

En el muro de la sala oeste se observa la traza de un arco de medio punto cegado que, probablemente, daría paso a otro espacio, inaccesible en la actualidad. A través de esta pared cae, a cierta altura, un chorro de agua de forma constante que constituye hoy el único aporte al aljibe. Esta filtración indica la existencia de bolsas de agua que, o bien manan allí, o se acumulan en esa zona a través de canalizaciones hoy olvidadas. La existencia de otros ejemplos conocidos en Toledo plantea la posibilidad de que esa zona pudiera corresponder a pozos excavados en la roca, origen, en muchas ocasiones, de la construcción de un aljibe ${ }^{10}$.

Estos aljibes se llenaban, además de con el agua de lluvia o de escorrentía superficial, con la que se captaba de aguas subterráneas por filtración, o con la que se traía de zonas alejadas por medio de canalizaciones ${ }^{11}$.

La recuperación de las aguas pluviales en edificios principales, a través de un complejo sistema de canalizaciones construidas en sus tejados, fue una solución que la arquitectura musulmana heredó de la romana y que, como veremos, se transmitió de igual forma a la arquitectura gótica ${ }^{12}$.

\section{El aljibe musulmán}

Las noticias que tenemos de la Mezquita Aljama de Toledo son pocas. De entre ellas destaca, en el último tercio del siglo IX, la autorización solicitada al emir para reconstruir su alminar caído, utilizando además para reedificarlo la sala de oración de una iglesia contigua ${ }^{13}$. La mezquita parece que se asentó sobre un edificio ante-

10 La excavación de estos pozos contribuía a controlar el nivel de la capa freática y reconducir el agua de la zona, evitando la humedad en el nuevo edificio y, además, suministraba piedra para su construcción. Debemos recordar que el solar donde se asienta la catedral es una zona en pendiente, una ladera ubicada entre varias corrientes de agua. Ejemplos de estos pozos toledanos excavados en la roca en: J.M. ROJAS RODRÍGUEZ-MALO y J.R. VILLA GONZÁLEZ, "Casas islámicas de Toledo", Entre el Califato y la Taifa: Mil años del Cristo de la Luz, Toledo, 1999, p. 208, y en J. PASSINI, Casas y casas principales urbanas. El espacio doméstico de Toledo a fines de la Edad Media, Madrid, 2004, pp. 468-470.

11 Actualmente, se pueden identificar tres grandes conducciones de época romana en dirección a la catedral. De noreste a sureste: la primera, desde las termas romanas de la Plaza Amador de los Ríos baja paralela a la calle del Nuncio Viejo; la segunda, baja desde las Cuevas de Hércules y, la tercera, de norte a sur, desde Cuatro Calles hacia la Puerta del Reloj. J. PASSINI, op. cit., 2004, p. 190.

12 La recuperación del agua de los tejados se hacía en la casa romana y se continuará en la arquitectura musulmana, así como la ubicación de los aljibes en el patio central. B. PAVÓN MALDONADO, op. cit., 1990, p. 15.

13 C. DELGADO VALERO, Toledo Islámico: Ciudad, Arte e Historia, Toledo, 1987, p. 266. 
rior, probablemente, una iglesia visigoda ${ }^{14}$. El estado del viejo edificio y el aumento del número de fieles, contribuyeron a tomar la decisión de ampliarla.

Además de los datos documentales, gracias al estudio que realizó Conrad von Konradsheim, midiendo la resistencia de los materiales en el subsuelo mediante el empleo de fuentes de corriente artificiales, conocemos un plano aproximado de la mezquita, su configuración y disposición ${ }^{15}$. El trazado conseguido a partir de este método corresponde a una sala de oración, de planta rectangular, ligeramente orientada al sureste y con el patio ocupando parte del claustro actual. Sus características formales y los motivos decorativos que se han conservado en la Catedral ayudaron a fijar su datación entre el siglo $\mathrm{X}$ y la primera mitad del siglo $\mathrm{XI}^{16}$ (fig. $1)$.

A los restos citados por Clara Delgado y al estudio de Konradsheim, podemos añadir los hallazgos arqueológicos de las recientes excavaciones realizadas en el claustro $^{17}$, y el gran aljibe construido para la Mezquita Aljama de Toledo ${ }^{18}$. Dos elementos, además de lo hasta ahora visto, confirman su filiación musulmana: los grafitos árabes grabados en el revestimiento de las paredes del aljibe ${ }^{19}$, y un brocal de pozo.

La huella donde estuvo colocado el brocal con que se remataba este aljibe, todavía se mantiene, y es visible desde el interior. Este espacio pervivió en la memoria del claustro, y se protegió con una escalera doble que salvaba el acceso al depósito $^{20}$, como se puede apreciar en una foto de Alguacil del siglo XIX.

14 Konradsheim identificó los basamentos de una primitiva iglesia de planta de cruz griega. G.C. KONRADSHEIM, "Exploration Géophysique des soubassements de la Cathédrale de Tolède", Annales d'Histoire de l'Art et d'Archeologie, Bruxelles, II (1980), pp. 95-99.

15 A pesar de sus lagunas, supuso el conocimiento más importante sobre la mezquita mayor de Toledo hasta las recientes excavaciones arqueológicas que, en gran medida, han confirmado su análisis. G.C. KONRADSHEIM, op. cit., 1980.

16 La profesora Clara Delgado cita las 55 piezas conservadas de la mezquita e incorporadas en la catedral: 54 fustes y un capitel; el capitel de la capilla de Santa Lucía y los fustes del trascoro que dató de época de Abd al-Rahman III. C. DELGADO VALERO, op. cit., 1987, p. 273.

17 Por primera vez se ha constatado arqueológicamente la existencia de la mezquita bajo la Catedral, y los hallazgos confirman sus dimensiones, y abundan en la datación del siglo XI. M. ALMAGRO-GORBEA, "Hallazgos arqueológicos en el subsuelo de la Catedral", La Catedral Primada de Toledo. Dieciocho siglos de Historia, Burgos, 2010, pp. 134-141.

18 En muchas ocasiones la función utilitaria de los aljibes los ha salvado, y a pesar de desaparecer los edificios para los que se construyeron, mantuvieron su uso como lugar de aprovisionamiento de agua. En la mayoría de los casos la datación cronológica de los aljibes es complicada por el uso de materiales reutilizados de diferentes épocas, y porque su continua utilización conlleva un mantenimiento que incluye limpieza y, en caso necesario, las reparaciones oportunas, obligando a una datación muchas veces relativa de estas obras. Sin embargo, en el caso del aljibe de la catedral de Toledo, la posterior construcción del claustro gótico sobre él lo mantuvo prácticamente inalterado, quedando protegida la obra islámica por la obra medieval.

19 M. ALMAGRO-GORBEA, op. cit., 2010.

20 El hueco del brocal está situado entre los dos pilares, en la bóveda baída de la sala oeste. El claustro adaptó su diseño a su presencia salvándolo con la construcción de la escalera doble que vemos en las fotos de Alguacil (M. GARCÍA RUIPÉREZ y R. DEL CERRO MALAGÓN, Toledo entre dos siglos en la Fotogra- 


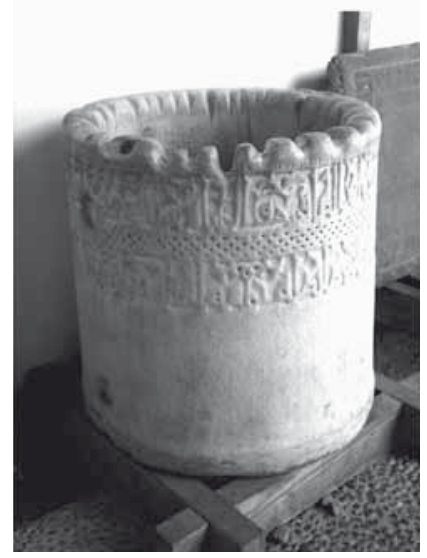

Fig. 2. Brocal de mármol blanco con inscripción que celebra el fin de la construcción del aljibe de la mezquita mayor de Toledo en el año 1032. (Museo de Santa Cruz, Toledo).

Un brocal de mármol blanco, que se conserva actualmente en el Museo de Santa Cruz, está decorado con una inscripción, en caracteres cúficos, en la que se celebra el fin de la construcción del aljibe de la Mezquita Mayor de Toledo en el año $1032^{21}$. Fue encargado por Al-Zahir, primer rey de la dinastía de los Du1-nuníes de Toledo. A mediados del siglo XVIII este brocal estaba colocado en el patio del Convento de San Pedro Mártir, donde Bayer lo dio a conocer ${ }^{22}$ (fig. 2).

La inscripción del brocal y las huellas que se mantienen en el claustro, y en el aljibe, nos conducen a proponer que pertenecía al aljibe del claustro de la Catedral, para el que se talló y con el que podemos datar su construcción en torno al año 1032.

El aljibe de la Mezquita Mayor de Toledo recibía también el agua que se recogía de los tejados ${ }^{23}$ y, quizás, de aportaciones que le llegaban a través de canalizaciones.

La cantidad de agua que almacenaba, y que después necesitaban elevar, era suficiente para tener que construir en el patio de la mezquita una noria que permitiera subir más de la que se podía sacar a través del pozo para el que tallaron el brocal de mármol. Identificamos la noria islámica en la abertura, de forma rectangular, que se sitúa en el muro norte de la sala oeste del aljibe, a la altura del arranque de la bóveda, hoy sólo visible desde el interior, al estar bajo los escalones del claustro que suben de la crujía al jardín ${ }^{24}$.

fía de Casiano Alguacil 1832-1914, Toledo, 2008, p. 117). El brocal se trasladó al Convento de San Pedro Mártir, donde lo fotografió Laurent y permaneció hasta 1872.

21 J. AMADOR DE LOS RÍOS, Toledo Pintoresca o Descripción de sus más celebres monumentos, Madrid, 1845, p. 231; M. MAROTO GARRIDO, Fuentes Documentales para el Estudio de la Arqueología en la Provincia de Toledo, Toledo, 1991, pp. 150-151; y F. DEL RÍO SÁNCHEZ, "Piedras para el Recuerdo. Un paseo por Toledo leyendo algunas inscripciones árabes”, Tulaytula, 3 (1998), pp. 21-29.

22 Biblioteca Capitular de Toledo, F. PÉREZ BAYER, De Toletano Hebraeorum templo, Ms. 1757, ff. 214v y $215 \mathrm{r}$.

${ }_{23}$ Para recoger el agua procedente de las cubiertas de la mezquita, tanto en la de Córdoba, como en la de Sevilla, recorrían la parte superior de cada nave caños de agua, a modo de acueductos. La Catedral de Sevilla. Fundación y fábrica de la obra nueva, Sevilla, 2007, pp. 220-228.

24 Las norias fueron muy abundantes en el Toledo medieval. En la descripción que del Toledo islámico hacen los geógrafos musulmanes la ciudad estaba rodeada de canalizaciones con norias para regar huertas y jardines, además de un acueducto de un arco sobre el Tajo, en cuya extremidad había una máquina hidráulica que subía el agua y, una vez arriba, la llevaba a la ciudad. C. DELGADO VALERO, "Noticias sobre Toledo suministradas por los geógrafos musulmanes", La España Medieval, t. V, Madrid, 1986, pp. 299-312. B. PAVÓN MALDONADO, "En torno al Acueducto y la Rueda Hidráulica Árabe de Toledo según Idrîsî”, AM, 5 (1997), pp. 273-293. 
En conclusión, podemos decir que en el primer tercio del siglo XI se construyó un gran aljibe en la Mezquita Aljama de Toledo para suministrar el agua necesaria al edificio religioso y a su barrio. Su situación sobre un eje, que no es totalmente paralelo al muro norte de la catedral (tiene una desviación aproximada de 3 grados), origina nuevas preguntas sobre la orientación de la planta de la mezquita aljama. Por otra parte, dos tapas más, situadas en la panda oeste del claustro, a la derecha de la puerta del Mollete, plantean la posibilidad de la existencia de otras salas del aljibe, o de pozos o cisternas anteriores a la propia mezquita.

\section{La Catedral y su noria gótica}

Tomada la ciudad por Alfonso VI, la mezquita, a pesar de que parece que fue otra la intención del rey, se consagró como iglesia. Tras cambiar su orientación y adaptarlo al culto cristiano, el edificio musulmán y sus construcciones anejas continuaron utilizándose. No fue hasta el primer tercio del siglo XIII, durante el arzobispado de don Rodrigo Jiménez de Rada, cuando se inicie la construcción del templo gótico y, aun así, el viejo edificio de la mezquita siguió en pie.

La construcción de la catedral se comenzó por la cabecera, y prosiguió por el colateral exterior sur de la nave, rodeando un obstáculo que, como describe Konradsheim, envolverá. Este obstáculo era la mezquita.

La obra avanzaba rápidamente ${ }^{25} \mathrm{y}$, a finales del siglo XIV, con el arzobispo don Pedro Tenorio se comenzó la construcción del claustro. La crujía este, entre la Puerta de Santa Catalina y la Capilla de San Blas, lugar elegido por el arzobispo para su enterramiento, fue la que se levantó en primer lugar. Se hizo a modo de nave independiente, como la parroquial de San Pedro que, posteriormente, y paralela al claustro, erigió el arzobispo Sancho de Rojas.

Durante el primer tercio del siglo XV se estaba trabajando en la Torre Mayor y en la Torre del Reloj ${ }^{26}$, y se proseguía la obra del claustro. La construcción de esta enorme fábrica requería gran cantidad de agua que, probablemente, suministraba el aljibe islámico, por lo que al mantener su funcionalidad continuó utilizándose sin apenas modificaciones.

Sin embargo, la construcción de los grandes pilares de la crujía sur provocó una serie de cambios. Mientras que el pozo y su brocal permanecieron en el mismo lugar, adaptándose el diseño del acceso al jardín para salvarlo, la noria islámica quedó inutilizada bajo el pavimento del claustro, y tuvo que construirse una nueva estructura rectangular fuera de la crujía, en el patio. Además, esta obra varió

25 Una revisión de las campañas constructivas en: T. NIKSON, "La Catedral: su Historia Constructiva", La Catedral Primada de Toledo. Dieciocho siglos de Historia, Burgos, 2010, pp. 148-161.

26 A.M. YUSTE GALÁn, "Puertas y Torres", La Catedral Primada de Toledo. Dieciocho siglos de Historia, Burgos, 2010, pp. 162-179. 
sustancialmente la superficie del depósito. Los basamentos de los tres pilares del claustro, ensanchados en sus bases para tener mayor solidez y sostener las altas bóvedas góticas, ocuparon casi un tercio del aljibe ${ }^{27}$.

La imposibilidad de seguir utilizando la noria islámica, y la necesidad de vaciar el aljibe, o mantenerlo por un tiempo con un nivel bajo, para poder asentar los pilares en el suelo del depósito debió ser la razón de la construcción de una noria gótica.

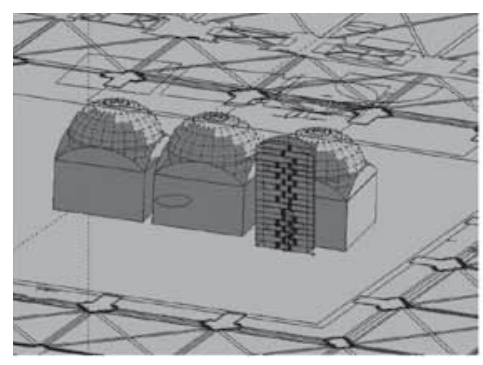

(1)

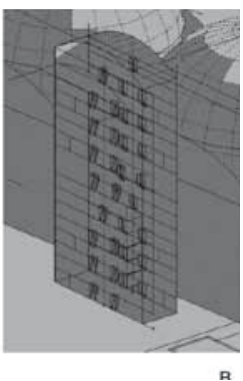

Fig. 3. A) Axonometría del aljibe y el pozo gótico. B) Detalle del pozo.

La comunicación del aljibe islámico con una cámara medieval, construida en paralelo, se realiza a través del tramo oeste del aljibe, donde se abre un estrecho e irregular hueco que accede a un pasillo, de aproximadamente metro y medio, por el que se llega a la cámara rectangular situada bajo el jardín del claustro (fig. 3).

La técnica con la que se construye esta estructura es diferente a la utilizada en el aljibe islámico, de muros de mampostería y bóvedas de ladrillo. La cámara gótica se construyó a base de gruesos sillares tallados en granito. Tiene una planta oblonga, de 3,26 x 1,03, una altura de 6,56 metros, y se cubre con una pequeña bovedilla. Desde ella la comunicación con el aljibe se hace a través de un gran vano, aquí perfectamente trazado, de 51 centímetros de ancho y 1,72 metros de alto, abierto en el eje de la estructura. Destaca su ejecución en grandes sillares ${ }^{28}$ dispuestos en hileras regulares, y asentados con un mortero mezclado con trozos de tejas quebradas de un espesor de unos dos centímetros ${ }^{29}$, y un revestimiento hidráulico, que sólo se conserva en la parte alta. En la parte baja, el mortero se ha perdido y permite observar directamente los sillares de granito con los que se construyó.

Muchos de los sillares conservan las marcas de cantero. Entre ellas predomina el triángulo, y se caracterizan por su gran tamaño, a diferencia de las que se han localizado en las crujías del claustro, con las que, por el momento, no se han podido relacionar.

27 La altura de las bóvedas del claustro va desde 10,50 a 10,90 metros. Sobre el suelo de los aljibes se puede observar la "suela" de los pilares medievales.

${ }^{28}$ Las medidas de algunos de estos sillares son: de largo 50, 60, 63, 66, 79 y $80 \mathrm{~cm}$. y, de altura, entre 32 y $33 \mathrm{~cm}$., con un modelo tipo de 66 x $32 \mathrm{~cm}$.

29 La peculiaridad de este mortero confiere al muro una mayor elasticidad para recibir la capa que impermeabilizará los paramentos. 
Además de su calidad técnica, llama la atención en esta construcción el aparejo de grandes ménsulas asentadas en los lados largos del rectángulo ${ }^{30}$. Estas ménsulas se disponen en nueve niveles, enfrentadas por parejas, alternando con una de mayor tamaño en cada lado. Están colocadas, de abajo a arriba, de la siguiente forma: tres niveles de parejas enfrentadas, el cuarto, sobre el dintel de la puerta, de una sola ménsula de mayor tamaño y centrada en cada lado, sobre ellas cuatro niveles más de parejas enfrentadas, para terminar con una ménsula centrada y de mayor tamaño a cada lado (fig. 4).

La forma y disposición de estas ménsulas sugieren que su finalidad era la de recibir una estructura de madera, posiblemente triangular, que formaría parte del engranaje de una máquina elevadora de agua $^{31}$.

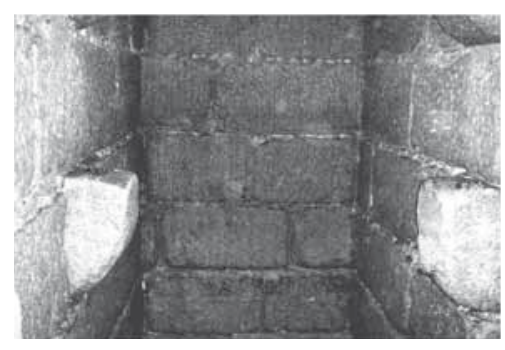

Fig. 4. Interior del pozo gótico. Detalle de las ménsulas.

Por todo ello, proponemos la identificación de esta cámara rectangular como el pozo de la noria gótica de la que se habla en la documentación medieval.

Durante el primer tercio del siglo XV el aljibe continuaba suministrando agua a la obra de la catedral, pero en estos momentos, como vimos, fue cuando la construcción del claustro afectó a la estructura de la noria islámica y obligó a desplazar hacia el jardín el pozo gótico. En 1418, como se recoge en la documentación de Obra y Fábrica de la Catedral de Toledo ${ }^{32}$, se estaba trabajando en el pozo de la huerta de la claustra y, excepcionalmente, aparece también citado su aljibe y las obras que se llevaban a cabo para que el agua de lluvia llegase hasta su depósito ${ }^{33}$.

Una vez construido el pozo, se encargó un ingenio para subir el agua a maestros carpinteros especialistas. La elección de la madera para su elaboración era una tarea compleja, utilizándose para ello maderas oscuras como la encina o el almez ${ }^{34}$. A pesar de ello, el roce continuo al que se veían sometidas las piezas obligaba a repararlas frecuentemente, apareciendo en las anotaciones de gastos numerosos asientos donde se recogen la compra de maderas, clavos, cuerdas, sebos para los cubos, cangilones..., elementos todos necesarios para mantener en buen uso la noria.

30 Las ménsulas sobresalen $13 \mathrm{~cm}$., tienen una anchura de $19 \mathrm{~cm}$., y la altura del sillar donde se tallan es de $32 \mathrm{~cm}$.

31 Como es habitual en los pozos de norias, su planta es rectangular para colocar la estructura de madera.

32 Los documentos pertenecientes al Archivo Capitular de Toledo (ACT), se citarán como O.F. (Obra y Fábrica) seguidos de su signatura correspondiente. O.F. 761, f. 96r: “... iohan gonçales e pero Rodrigues alvañiles que labro el uno este dia en las casas de Alvar martines maestro e el otro en el pozo de la huerta de la claustra: 20 cada uno...".

33 ACT, O.F. 761, f. LXXVIIIr y f. 36r. Véase nota 42.

34 J. GONZÁLEZ TASCÓN, “Agua para ciudades y Regadíos”, Ars mechanicae. Ingenieria medieval en españa, Madrid, 2008, p. 79. 
Los gastos por reparaciones eran continuos, hasta que no quedaba más remedio que hacer ruedas nuevas o incluso una noria nueva, como ocurrió en 1424. Durante este año se compraron madera de encina, maderos de álamo negro "al maestro de hacer norias", caranis medianos (clavos) y numerosas piezas de hierro al herrero ${ }^{35}$. A finales de noviembre de 1424 los carpinteros asentaron la noria nueva en el pozo del claustro ${ }^{36}$.

En 1431 se anotó el pago por una bestia y el mozo que la arreaba para sacar agua del pozo de la noria ${ }^{37}$. Parece que la nueva noria que instalaron era, probablemente, una noria de sangre o saqiya, noria de tracción animal de uso generalizado durante la Edad Media ${ }^{38}$.

35 ACT, O.F. 762, f. 91v: “... para faser el anoria e açeña para sacar Agua del pozo de la claustra para la huerta della... 20 palancas de hensina para faser el anoria del pozo de la claustra...”; f. 92v: “... Miercoles 14 de junio costaron quatro maderos de alamo negral para faser una Rueda para el anoria del pozo de la claustra 60 maravedis el qual se conpro de un carpintero que mora a sant viçente que fase anorias... tablas para hacer un anaquel para donde caya el agua del anoria del pozo de la claustra 50 maravedis...”; f. 94r: “... una vara de pino para calar la zebratana del anoria del pozo..."; f. 94v: “...viernes 27 de otubre se pago de 156 maravedis que ovo de aver de 3 carretadas de madera de Alamo para la anoria de la huerta de la claustra... a 52 maravedis cada carretada...sabado 21 de otubre... nueve palancas de ensina para faser çegumelas para las canpanas... otrosy para el anoria del pozo de la huerta 19 maravedis ... 70 torçeros de madera que se tornaron de grandes pequeños para la Rueda del anoria de la claustra... los quales fiso iohan Ruys tornero veçino de Toledo..... 70 capatillas de cuero coradas para los dichos torteros de la dicha anoria...martes 28 ... costo un madero de alamo negral seco que se conpro para el anoria... 100 maravedis"; f. 97v: “... 622 maravedis de çiertas obras que fiso e dio para la Anoria Nueva que se fase para la huerta de la claustra para sacar agua del pozo... dio çinco aguijones et un palo et ajuelo de fierro Nuevos que pesaron 82 libras... dio 25 libras e media de gorrones... 9 sorajas de fierro que pesaron 48 libras.... son todas 155 libras e media a quatro maravedis la libra que montaron los dichos 622 maravedis"; f. 98v: "34 libras e media de perlos con sorajas e chavetas para el Anoria Nueva de mudar que se fizo para el pozo de la huerta de la claustra A çinco maravedis la libra que son 672 maravedis... dio mas un fierro de çepillo que mando faser por andres maestro para la zebriana del pozo de la huerta...por 50 maravedis... sabado 10 de Junio... pago a Ferrand garçia haron ferrero vesino de toledo 268 maravedis que ovo de aver por rrason de dos sorajas de fierro e çiertos fusillos de fierro que dio e fiso par la obra de las Ruedas del pozo del huerta de la claustra...”; f. 99r: “...lunes 3 dias de Jullio... pago a ferrand garçia ferrero veçino de toledo 28 maravedis que ovo de aver de siete libras de plegaja e sorajas que avia dado para la anoria arrason de 4 maravedis la libra...."; f. 100r: “... martes 31 de otubre... dos aguijones de fierro que se fisieron para el anoria del pozo de la claustra que pesaron 13 libras cada a 4 maravedis la libra que son $53 \ldots$ Item costaron tachuelas que fueron menester para la obra de la dicha anoria 15 maravedis que son todos 38 maravedis... Jueves 2 de Noviembre... pago a Johan gonçales de esquivias ferrero vesino de toledo 92 maravedis del fierro para la anoria de la claustra: dos sorajas grandes 30 maravedis. Item una soraja que adobo para el exe mayor que la torno quadrada et otras dos que torno e fiso de rredondas quadradas para el exe menor de la dicha anoria... ferrete para foradar e un açel para cortar clavos e çapatas... 8 lañas para la rueda de la anoria..."; f. 133v: “... carpinteros van a cortar madera de alamos negrales para faser la noria et artifiçio para sacar agua del pozo de la huerta de la claustra...”; f. 143v: “... Johan sanches odrero que anduvo con pero andres a pegar los cangilones... e obra de la noria... 20 maravedis...".

36 ACT, O.F. 762, f. 144v: “... Martes 30... andamios donde se a de sentar la noria...”.

37 ACT, O.F. 768, f. 127v.

38 Sobre el origen, evolución y partes de la noria véase: J. CARO BAROJA, "Sobre la historia de la noria de tiro", Tecnología Popular Española, Madrid, 1996, pp. 337-392; M. BARCELÓ, H. KIRCHNER y C. NAVARRO, El agua que no duerme. Fundamentos de la arqueología hidráulica andalusí, Granada, 1996, pp. 279-294; y J. GONZÁLEZ TASCÓN, op. cit., 2008, p. 73. 
Cuando el diámetro del pozo lo permitía, se instalaba este tipo de noria de tracción animal que, movida por bestias de tiro, permitía sacar una mayor cantidad de agua. La noria de sangre, se compone de dos grandes ruedas: una horizontal, denominada arbolote, que es la que mueve el animal y transmite su giro a otra vertical, la rueda de agua, instalada sobre la boca del pozo, que lleva una cuerda circular ${ }^{39}$ con los cangilones o alcaduces atados que cuelgan hasta el fondo del pozo y que al girar la rueda se llenan subiendo el agua a la superficie.

Desde 1424 a 1467 aparecen numerosas anotaciones por la compra y reparación de piezas para la noria, y de su continuo uso suministrando el agua necesaria para las obras de la catedral, y para regar la huerta que, en esos años, crecía en el jardín ${ }^{40}$.

Cipreses, arrayanes y laureles, traídos del Monasterio de Santa María de Guadalupe se plantaron en el jardín del claustro de la Catedral de Toledo y se protegieron para que no se dañasen ${ }^{41}$.

El edificio gótico también heredó la solución empleada de recuperación del agua de lluvia en los tejados para llenar el aljibe. En 1418 este sistema de recogida de agua estaba en funcionamiento con las obras de reparación y mantenimiento necesarias. Se limpiaban las gárgolas, se abrían canales y se construían arcas para recibir el agua y llevarla al aljibe ${ }^{42}$.

De esta compleja red para recoger y conducir el agua de los tejados quedaban todavía restos a mediados del siglo XIX. Parro describe conductos "con mucha inteligencia construidos" para que el agua de los tejados vertiera en diferentes pun-

39 La elaboración de las cuerdas empleadas para este fin era una tarea compleja llevada a cabo por varias personas. Se anotan muchos gastos de sogas donde se atan los alcaduces de la noria. ACT, O.F. 768, ff. 45r, 103v, 116r; ACT, O.F. 769, ff. 49r y v; ACT, O.F. 774, f. 60v; ACT, O.F. 775, f. 72v; y ACT, O.F. 782, f. $24 \mathrm{r}$.

40 ACT, O.F. 763, f. 60r; ACT, O.F. 767, f. 64r; ACT, O.F. 768, ff. 131v y 134v; ACT, O.F. 769, ff. 99r, 120r, 121v, 132 r y v; ACT, O.F. 777, f. 112r; y ACT, O.F. 782, f. 24v.

${ }^{41}$ ACT, O.F. 763, ff. 37r y 56r: “... 14 de junio, 21 varas de pino que se compraron para la huerta de la claustra para los cipreses e laureles e otros árboles de ella...”. En 1427 hay problemas de agua, los pozos de la claustra se secaron y fue necesario comprar cargas de agua para regar los árboles de la huerta, aun así poco después trajeron de nuevo árboles de Guadalupe para plantar. ACT, O.F. 765, ff. 38r, 129r y 131v.; ACT, O.F. 768, f. 60v: “... se compran 20 libras de vellote mediano para çercar la huerta de la claustra con los Rollizos para que no entrasen en la huerta..."; f. 111v: ... 4 carpinteros que labraron en los andores de los paños de la claustra para çercar que no entren a la huerta los que juegan a la pellota e lo çercaron de Rollizos de pino con su clavazon...".

42 ACT, O.F. 761, f. LXXVIIIr: "cuatro peones e una muger que çernyeron e amasaron cal e cavaron e sacaron tierra de la capilla de sant Estevan para faser una arca donde se acogiese el agua de la luvya et vinyese al algibe..."; f. 36r: “... Item fizo mas quatro rrailes para que entre el agua limpia al algibe de la claustra...”; f. 106r: “... este dia anduvieron 4 peones que anduvieron alinpiando las gargolas de las 4 naves de la claustra para que estovyesen linpias por donde vinyese el agua a salir fuera de los tejados a dies cada uno...". ACT, O. F. 763, f. 41r: “...Atunes pozo de la clavería”; f. 41v: “... alcaduçes grandes para los caños de los tejados de la iglesia por donde vienen a las gargolas el agua de los tejados... Guindaleta para la noria del pozo de la huerta de la claustra...". 
tos. Alguno de estos conductos estaban colocados en el grueso de pilares y columnas, y el sistema comunicaba además con dos grandes alcantarillas de la ciudad ${ }^{43}$.

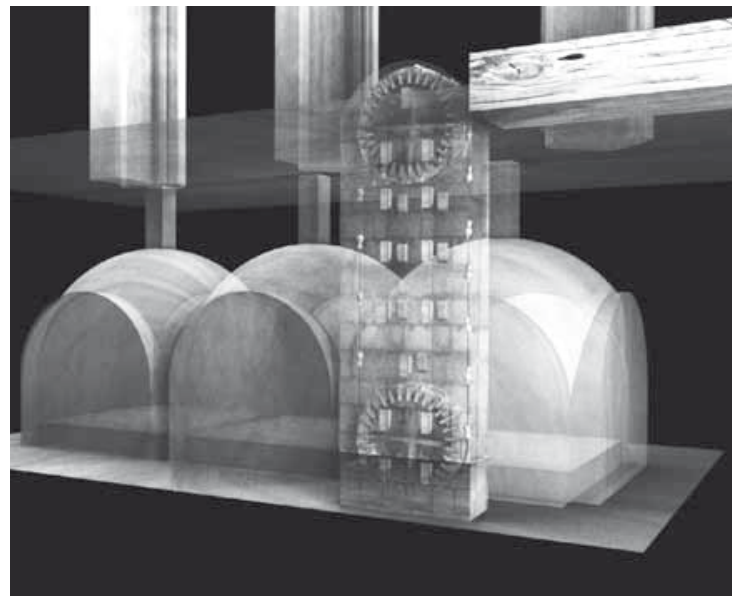

Fig. 5. Restitución de la noria gótica y el aljibe musulmán. (Dibujo de Matías Capuchino).
Esta infraestructura hidráulica se completa con dos estanques, o albercas, situados en el jardín del claustro (que se mantienen aunque reformados), colocados a ambos lados de la noria gótica ${ }^{44}$. Su función sería, por una parte, recibir el agua que se subía con la noria y, por otra, el agua de los tejados que caía de las gárgolas, y que podrían almacenar hasta bajarla al aljibe, con el que estaban conectados a través del pozo de la noria. Ambos estanques aparecen en un plano de 1604 de la catedral ${ }^{45}$.

\section{Conclusión}

El aljibe que se encuentra bajo la panda sur del claustro pertenecía a la Mezquita Mayor de Toledo y ha pervivido hasta nuestros días formando parte de la Catedral que lo mantuvo y lo siguió utilizando. Todos los edificios fueron dejando una huella más o menos perceptible en la traza y en el espacio de la siguiente construcción. En el ejemplo que nos ocupa, el maestro que trazó el diseño del claustro asumió la funcionalidad del aljibe islámico y los pedreros medievales continuaron, ante el reto que suponía el suministro del agua, empleando soluciones heredadas, adaptándolas a sus necesidades y aplicando las nuevas técnicas constructivas para elevar el pozo de una noria gótica (fig. 5).

43 S.R. PARRO, Toledo en la Mano, Toledo, 1857, t. I, p. 69

44 ACT, O.F. 772, ff. 163r, 164 v y 171 v: “... Aparejador más 21 maestros... piedra berroqueña para el pavimento e alberca que se fase en la claustra...".

${ }^{45}$ Libro de la Fundación de la S. I. de Toledo, publicado en: R. GONZÁLVEZ y F. PEREDA, La Catedral de Toledo. 1549. Según el Dr. Blas Ortiz. Descripcion Graphica y Elegantissima de la S. Iglesia de Toledo, Toledo 1999, pp. 148-149. 